\title{
Platelet activation in critically ill COVID-19 patients
}

Nader Yatim ${ }^{1,2+} \mathbb{C}^{0}$, Jeremy Boussier ${ }^{1+}{ }^{\circledR}$, Richard Chocron ${ }^{12,14}$, Jérôme Hadjadj 2,3 , Aurélien Philippe ${ }^{4,5,6}$, Nicolas Gendron 4,5,6, Laura Barnabei ${ }^{3}$, Bruno Charbit', Tali-Anne Szwebel ${ }^{2}$, Nicolas Carlier ${ }^{8}$, Frédéric Pène ${ }^{9,10}$, Célia Azoulay ${ }^{2}$, Lina Khider ${ }^{6,11}$, Tristan Mirault ${ }^{11,12}$, Jean-Luc Diehl ${ }^{6,8,13}$, Coralie L. Guerin ${ }^{4}$, Frédéric Rieux-Laucat ${ }^{3}$, Darragh Duffy ${ }^{1,7}$, Solen Kernéis ${ }^{15,16,17}$, David M. Smadja ${ }^{4,5,6+}$ and Benjamin Terrier ${ }^{2,12,18^{*}+}$

\begin{abstract}
Background: Microvascular, arterial and venous thrombotic events have been largely described during severe coronavirus disease 19 (COVID-19). However, mechanisms underlying hemostasis dysregulation remain unclear.

Methods: We explored two independent cross-sectional cohorts to identify soluble markers and gene-expression signatures that discriminated COVID-19 severity and outcomes.

Results: We found that elevated soluble (s)P-selectin at admission was associated with disease severity. Elevated sPselectin was predictive of intubation and death ( $R O C A \cup C=0.67, p=0.028$ and $A \cup C=0.74, p=0.0047$, respectively). An optimal cutoff value was predictive of intubation with $66 \%$ negative predictive value (NPV) and $61 \%$ positive predictive value (PPV), and of death with 90\% NPV and 55\% PPV. An unbiased gene set enrichment analysis revealed that critically ill patients had increased expression of genes related to platelet activation. Hierarchical clustering identified ITG2AB, GP1BB, PPBP and SELPLG to be upregulated in a grade-dependent manner. ROC curve analysis for the prediction of intubation was significant for SELPLG and PPBP (AUC $=0.8, p=0.046$ for both). An optimal cutoff value for PBPP was predictive of intubation with 100\% NPV and 45\% PPV, and for SELPLG with 100\% NPV and 50\% PPV.
\end{abstract}

Conclusion: We provide evidence that platelets contribute to COVID-19 severity. Plasma sP-selectin level was associated with severity and in-hospital mortality. Transcriptional analysis identified PPBP/CXCL7 and SELPLG as biomarkers for intubation. These findings provide additional evidence for platelet activation in driving critical COVID-19. Specific studies evaluating the performance of these biomarkers are required.

Keywords: COVID-19, Primary hemostasis, Platelets, Thrombo-inflammation

\section{Background}

Severe acute respiratory syndrome coronavirus 2 (SARS$\mathrm{CoV}-2)$ is the causative agent of the coronavirus disease 2019 (COVID-19) pandemic [1]. Severe disease is characterized by an acute respiratory distress syndrome

\footnotetext{
*Correspondence: benjamin.terrier@aphp.fr

${ }^{\dagger}$ Nader Yatim and Jeremy Boussier have contributed equally to this work

${ }^{\dagger}$ David M. Smadja and Benjamin Terrier are co-senior authors

${ }^{2}$ Department of Internal Medicine, National Reference Center for Rare

Systemic Autoimmune Diseases, AP-HP, APHP.CUP, Hôpital Cochin,

75014 Paris, France

Full list of author information is available at the end of the article
}

(ARDS), respiratory failure and death in about $1 \%$ of cases $[2,3]$. Most important risk factors for severe disease include age, overweight, diabetes, hypertension and history of cardiovascular disease $[4,5]$. Severe and critical patients were shown to develop arterial and venous thrombotic complications, such as pulmonary embolism, stroke and myocardial infarction [6, 7]. Markers of coagulation activation, in particular increased D-dimer, fibrinogen and von Willebrand factor (vWF) levels were found to be associated with critical illness, whereas only minor changes were noted in prothrombin time and platelet counts [8-11]. In addition, autopsy series 
described multiple thrombosis in deceased COVID-19 patients $[12,13]$. These findings suggest vascular microthrombotic disease as a primary factor for mortality in critically ill COVID-19 [6, 14]. Therefore, some authors supported the systematic use of curative anticoagulation upon admission to intensive care unit (ICU) [15], a strategy that has been reported to decrease mortality in severe COVID-19 [8, 16-18].

Mechanisms underlying increased thrombotic events are still unclear but accumulating evidence point to a key role for endothelial and platelet activation [19, 20]. As viral inclusions were described in endothelial cells, it has been hypothesized that endothelial cell injury and activation could drive platelet activation and subsequent coagulopathy $[13,21]$. Therefore, dissecting the contribution of platelets to COVID-19 critical illness is key to understand SARS-CoV-2 infection pathogenesis and identify novel therapeutic strategies.

Platelet P-selectin is a key thromboinflammatory molecule involved in platelet activation and function. It has been demonstrated to play a crucial role in primary hemostasis by regulating platelet-leukocyte interactions, fibrin and tissue factor recruitment into platelet aggregates and thrombus formation [22]. Its soluble form, sPselectin, is released upon platelet and/or endothelial cell activation and measurement of sP-selectin has been proposed as a reliable marker of in vivo platelet activation [23]. Moreover, sP-selectin levels have been shown to correlate with acute lung injury severity score and related death [24].

The aim of our study was to assess, in hospitalized COVID-19 patients, the ability of sP-selectin to predict requirement for mechanical ventilation and in-hospital mortality. Next, using whole-blood transcriptional data, we uncovered a platelet activation transcriptional signature associated with critical illness.

\section{Methods \\ Cohorts}

Two independent cohorts were analyzed for this study. Data used for the analysis of soluble P-selectin's ability to predict admission to ICU were extracted from a noninterventional study that was conducted at European Georges Pompidou Hospital (Paris, France) and partially described in [25] (Cohort 1). Briefly, Cohort 1 included consecutive patients with SARS-CoV-2 infection. Inclusion criteria were patients over 18 years of age, with a proven SARS-CoV-2 infection, which presented to the emergency department with hospitalization criteria. Patients were then hospitalized into conventional wards or directly to the ICU. For all patients, baseline characteristics (demographic, treatment, clinical, cardiovascular risk factors and body mass index) and biological data were retrieved from the medical records using a standardized data collection. As a control non-COVID-19 septic ICU cohort, we included plasma samples from a previously described pre-pandemic cohort $[25,26]$.

Cohort 2 [27] was conducted between March 19, 2020 and April 3, 2020 in Cochin Hospital (Paris, France), in the setting of the local RADIPEM biological samples collection derived from samples collected in routine care. Inclusion criteria for COVID-19 inpatients were: age between 18 and 80 years, diagnosis of COVID-19 according to WHO interim guidance and positive SARS-CoV-2 RT-PCR testing on a respiratory sample (nasopharyngeal swab or invasive respiratory sample). Detailed clinical and immunological characterization of the cohort was previously described in [27]. Epidemiological, demographic, clinical, laboratory, treatment, and outcome data were extracted from electronic medical records using a standardized data collection form.

The severity of COVID-19 was classified at the time of inclusion based on the adaptation of the Sixth Revised Trial Version of the Novel Coronavirus Pneumonia Diagnosis and Treatment Guidance and described in [27].

\section{Soluble P-selectin measurement}

sP-selectin quantification was performed on Cohort 1. Plasmas were collected on $0.129 \mathrm{M}$ trisodium citrate tubes (9NC BD Vacutainer, Plymouth, UK). Plasma poor platelet was obtained after centrifugation twice at $2500 \mathrm{~g}$ for $15 \mathrm{~min}$. PPP was frozen after a second centrifugation at $2500 \mathrm{~g}$ for $15 \mathrm{~min}$ and stored at $-80^{\circ} \mathrm{C}$ until analysis of vascular markers. Soluble P-selectin were quantified in PPP with a Human Magnetic Luminex Assay from R\&D systems (Lille, France). Data were assessed with the Bio-Plex 200 using the Bio-Plex Manager 5.0 software (Bio-Rad, Marnes-la-Coquette, France). Normalized concentration $(\mathrm{NC})$ used to calculate receiver operating characteristic (ROC) area under curve (AUC) $p$ values represents sP-selectin concentration in $\mathrm{pg} / \mathrm{mL}$ normalized to platelet numbers $\left(10^{6} / \mathrm{mL}\right)$.

\section{Gene expression analysis}

Detailed methods was previously reported in Hadjadj et al. [27]. Briefly, we analyzed $100 \mathrm{ng}(5 \mu \mathrm{L})$ of total RNA from each sample using the Nanostring Human Immunology kit v2 according to manufacturer's instructions. Raw RNA counts were adjusted using five housekeeping genes selected from the 15 candidate control genes provided by Nanostring, following the geNorm method. For gene set enrichment analysis (GSEA), genes were ordered by $t$-statistic from unpaired $t$ test comparing normalized RNA levels of severe vs critical patients, and then fed to the gene set enrichment algorithm (GSEA version 4.0.3, Broad Institute), along with a pathway data set built 
from the Nanostring Immunology panel version 2 annotation file. Parameters were set as follows: method, pre ranked gene list; number of permutations, 2000; enrichment statistic, classic; min set size, 5; max set size, 200; and all other parameters as default. Hierarchical clustering of genes belonging to the hemostasis gene set was performed using hclust with default distance matrix. Heatmap displaying genes that are upregulated in a grade-dependent manner was obtained using pheatmap (package pheatmap), with data centered to 0 and scaled to unit variance for each gene. Normalized RNA count (NRC) used to calculate ROC AUC $p$ values represents adjusted RNA count (to housekeeping genes) normalized to platelet numbers $\left(10^{9} / \mathrm{L}\right)$.

\section{Statistical analyses}

Quantitative variables were compared among groups using Kurskal-Wallis test followed by Dunn's post-test, while quantitative variables were compared using the $\mathrm{X}^{2}$ test of independence. Correlations coefficients and $p$ values were assessed using Spearman's method. ROC AUC $p$ values were determined using Hanley's method. Optimal thresholds were determined by maximization of Youden's index. Multivariate logistic regression was performed using death as the dependent variable, and $\log _{10}$ (normalized P-selectin), age and sex as independent variables. All analyses were two-sided and a $p$ value smaller than 0.05 was considered statistically significant. Statistical analyses were performed using R v. 3.4.3 (CRAN).

\section{Results}

\section{Patient characteristics}

Cohort 1 consisted of 60 COVID-19 patients that were admitted to European Georges Pompidou Hospital and included upon admission. Clinical and biological characteristics are previously reported in [28] and described in Table 1. Briefly, median age was 58.5 years (IQR, 49 to 72 ) and $76.7 \%$ were male. Patients were analyzed after a median duration of 6 days (IQR, 4 to 7) after onset of first symptoms. Interval from first symptoms on hospital admission and from first symptoms on blood sampling coincided in most patients. Fever was present in $97 \%$ of the patients, and other most common symptoms were cough $(83.3 \%)$, dyspnea $(71.7 \%)$, fatigue $(70.0 \%)$, myalgia (40.0\%) and diarrhea (26.7\%). Degree of COVID19 severity was categorized as mild-to-moderate in 35 patients (clinical symptoms associated with dyspnea and requiring a maximum of $3 \mathrm{~L} / \mathrm{min}$ ), severe in 10 patients (respiratory distress requiring more than $3 \mathrm{~L} / \mathrm{min}$ of oxygen and no other organ failure) and critical in 15 patients (respiratory failure requiring mechanical ventilation). Eleven out of 35 patients with mild-to-moderate disease and 3/10 patients with severe disease experienced clinical worsening and required mechanical ventilation. Thrombotic events were noted in 8 patients ( 3 mild-tomoderate, 2 severe and 3 critical). We also included and analyzed plasma samples from a previously described pre-pandemic cohort $[25,26]$ as an ICU control nonCOVID-19 septic ICU cohort.

Cohort 2 consisted of 50 patients with various degree of COVID-19 severity admitted to Cochin hospital and 18 healthy controls, and was described in [27]. For the purpose of the present study, only patients with wholeblood transcriptional analysis were included, i.e., 32 COVID-19 patients and 13 healthy controls. Characteristics of the patients are described in Table 2. Median age was 56 years (IQR, 51 to 65 ) and $78 \%$ were male, while median age of healthy controls was 59 years (IQR, 41 to 60 ) and $77 \%$ were male. Patients were analyzed after a median duration of 10 days (IQR, 9 to 11) after onset of first symptoms. Interval from first symptoms on hospital admission and from first symptoms on blood sampling coincided in most patients. Fever was present in $100 \%$, and other most common symptoms were dyspnea $(100 \%)$, fatigue $(32 \%)$, cough $(97 \%)$, myalgia $(97 \%)$ and diarrhea (34\%). Degree of COVID-19 severity was categorized as mild-to-moderate in 11 patients (median oxygen requirement $1.5 \mathrm{~L} / \mathrm{min}$ ), severe in 10 patients (median oxygen requirement $5 \mathrm{~L} / \mathrm{min}$ ) and critical in 11 patients. No patients with mild-to-moderate disease required admission to an ICU, while 5 out of 10 patients with severe disease were eventually admitted to the ICU. Thrombotic events were noted in 3 patients ( 1 severe and 2 critical).

\section{Increased plasma sP-selectin on hospital admission is associated with disease severity and in-hospital mortality}

We measured plasma sP-selectin in Cohort 1. Increased plasma sP-selectin levels at admission was significantly associated with critical disease (Fig. 1a) in COVID19 patients, albeit at similar levels compared to nonCOVID-19 septic ICU patients (Fig. 1a and [25, 26]). Moreover, sP-selectin levels were positively correlated with inflammatory parameters, i.e., CRP levels $(r=0.30$, $p=0.017$, Fig. $1 \mathrm{~b})$, but not with platelet count $(r=0.1$, $p=0.43$, Fig. $1 \mathrm{c}$ ).

We next evaluated the ability of sP-selectin, normalized to platelet counts, to predict intubation (Fig. 1d) or inhospital mortality (Fig. 1e) using ROC curves. We found that outcomes were significantly associated with higher sP-selectin values $(\mathrm{AUC}=0.67, p=0.028$ for intubation and $\mathrm{AUC}=0.74, p=0.0047$ for in-hospital mortality). An optimal cutoff value of 150 normalized concentration $\left(\mathrm{pg} / \mathrm{mL}\right.$ normalized to $10^{6}$ platelets $\left./ \mathrm{mL}\right)$ was predictive 
Table 1 Clinical characteristics and laboratory findings of patients from Cohort 1

\begin{tabular}{|c|c|c|c|c|c|}
\hline \multirow[t]{3}{*}{ Characteristics } & \multirow{3}{*}{$\begin{array}{l}\text { All patients } \\
N=60\end{array}$} & \multicolumn{3}{|l|}{ Disease severity } & \multirow[t]{3}{*}{$p$ value } \\
\hline & & Mild-to-moderate & Severe & Critical & \\
\hline & & $N=35$ & $N=10$ & $N=15$ & \\
\hline Age, median (IQR), year & $58.50[49.00,72.25]$ & $59.00[47.50,74.00]$ & $58.00[47.25,70.00]$ & $58.00[54.00,68.50]$ & 0.98 \\
\hline Male, no. (\%) & $46(76.7)$ & $22(62.9)$ & $10(100.0)$ & $14(93.3)$ & 0.011 \\
\hline Median interval from first symptoms & $6.0[4.0,8.0]$ & $6.0[3.0,7.0]$ & $4.0[4.0,7.5]$ & $7.0[6.5,9.0]$ & 0.063 \\
\hline \multicolumn{6}{|l|}{ Coexisting disorder, no. (\%) } \\
\hline Any & & $18(51.4)$ & $5(50.0)$ & $11(73.3)$ & 0.18 \\
\hline COPD & $1(1.7)$ & $0(0.0)$ & $0(0.0)$ & $1(6.7)$ & 0.21 \\
\hline Diabetes & $16(26.7)$ & $8(22.9)$ & $3(30.0)$ & $5(33.3)$ & 0.72 \\
\hline Hypertension & $27(45.0)$ & $14(40.0)$ & $5(50.0)$ & $8(53.3)$ & 0.65 \\
\hline Cardiovascular disease & $12(20.0)$ & $5(14.2)$ & $3(30.0)$ & $4(26.7)$ & 0.26 \\
\hline Cancer or hemopathy & $3(5.0)$ & $3(8.6)$ & $0(0.0)$ & $0(0.0)$ & 0.32 \\
\hline Chronic renal disease & $8(13.3)$ & $4(11.4)$ & $3(30.0)$ & $1(6.7)$ & 0.21 \\
\hline Overweight & $39(65.0)$ & $19(54.3)$ & $7(70.0)$ & $13(86.7)$ & 0.026 \\
\hline \multicolumn{6}{|l|}{ Fever on admission } \\
\hline Median temperature $(\mathrm{IQR}),{ }^{\circ} \mathrm{C}$ & $38.20[37.70,38.80]$ & $38.20[37.80,38.70]$ & $38.00[37.60,38.35]$ & $38.70[37.40,39.00]$ & 0.53 \\
\hline \multicolumn{6}{|l|}{ Symptoms on admission no. (\%) } \\
\hline Fever & $58(96.7)$ & $34(97.1)$ & $9(90.0)$ & 15 (100) & 0.38 \\
\hline Cough & $50(83.3)$ & $32(91.4)$ & $7(70.0)$ & $11(73.3)$ & 0.14 \\
\hline Dyspnea & $43(71.7)$ & $21(60.0)$ & $8(80.0)$ & 15 (100) & 0.046 \\
\hline Fatigue & $42(70.0)$ & $21(60.0)$ & $8(80.0)$ & 15 (100) & 0.36 \\
\hline Myalgia & $24(40.0)$ & 15 (42.9) & $4(40.0)$ & $5(33.3)$ & 0.87 \\
\hline Diarrhea & $16(26.7)$ & 10 (28.6) & $3(30.0)$ & $3(20.0)$ & 0.79 \\
\hline Median oxygen requirement (IQR, L/min) & $3.00[2.00,5.00]$ & $2.00[1.25,2.00]$ & $4.00[4.00,5.00]$ & MV & - \\
\hline \multicolumn{6}{|l|}{ Clinical outcomes, no. (\%) } \\
\hline Thrombotic event (\%) & $8(13.3)$ & $3(8.6)$ & $2(20.0)$ & $3(20.0)$ & 0.44 \\
\hline Clinical worsening requiring MV (\%) & - & $11(31.4)$ & $3(30.0)$ & - & - \\
\hline Death (\%) & $17(28.3)$ & $4(11.4)$ & $2(20.0)$ & $11(73.3)$ & $<0.001$ \\
\hline \multicolumn{6}{|l|}{ Laboratory findings on admission } \\
\hline Leukocytes (IQR), × 109 /L & $5.75[4.47,7.77]$ & $5.40[4.15,6.85]$ & $6.35[5.05,10.92]$ & $7.60[4.90,11.90]$ & 0.041 \\
\hline Neutrophils (IQR), $\times 10^{9} / \mathrm{L}$ & $4.36[3.13,6.83]$ & $3.74[2.84,5.18]$ & $5.43[3.89,10.86]$ & $6.99[3.88,10.48]$ & 0.015 \\
\hline Lymphocytes (IQR), × 109 /L & $0.85[0.62,1.15]$ & $0.88[0.71,1.10]$ & $0.85[0.57,1.25]$ & $0.60[0.46,1.05]$ & 0.19 \\
\hline Monocytes (IQR), $\times 10^{9} / \mathrm{L}$ & $0.35[0.24,0.49]$ & $0.35[0.25,0.48]$ & $0.43[0.35,0.59]$ & $0.26[0.16,0.53]$ & 0.26 \\
\hline Platelets (IQR), × 10% /L & $164[124,221]$ & $165[124,216]$ & $167[135,242]$ & $162[127.00,258]$ & 0.79 \\
\hline CRP (IQR), mg/L & $104[62,168]$ & $86[38,120]$ & $117[109,184]$ & $161[98,207]$ & 0.005 \\
\hline Lactate dehydrogenase (IQR), U/L & - & - & - & - & \\
\hline Fibrinogen (mean (SD)), g/L & $5.83[4.90,6.50]$ & $5.30[4.62,6.30]$ & $6.80[5.85,7.38]$ & $6.30[5.65,7.00]$ & 0.006 \\
\hline DDimer (mean (SD)), ng/ml & $999[662,1724]$ & $789[537,1270]$ & $1145[759,2142]$ & $1170[988,2151]$ & 0.05 \\
\hline Soluble Pselectine, pg/mL & $23663[18693,30394]$ & $20556[17484,29677]$ & $25239[21637,28565]$ & $27041[24171,38934]$ & 0.048 \\
\hline
\end{tabular}

Significant values $(p<0.05)$ are emphasized in bold

Quantitative variables are described as median (interquartile range) and compared using the Kruskal-Wallis test; qualitative variables are described as $n$ (\%) and compared using the $X^{2}$ test of independence

$C O P D$ chronic obstructive pulmonary disease, $C R P C$-reactive protein, $I Q R$ interquartile range, $M V$ mechanical ventilation

for intubation with $66 \%$ sensitivity, $61 \%$ specificity, $66 \%$ negative predictive value (NPV) and $61 \%$ positive predictive value (PPV), and death with $82 \%$ sensitivity, $60 \%$ specificity, 90\% NPV and 55\% PPV. Multivariate logistic regression after adjustment for age and gender confirmed the association of elevated sP-selectin with in-hospital mortality $(\mathrm{OR}=72.0 \quad(2.99-3600.00), p=0.016)$. Clinical follow-up of COVID-19 patients initially non-critical 
Table 2 Clinical characteristics and laboratory findings of patients from Cohort 2

\begin{tabular}{|c|c|c|c|c|c|c|}
\hline \multirow[t]{3}{*}{ Characteristics } & \multirow{3}{*}{$\begin{array}{l}\text { Healthy controls } \\
N=13\end{array}$} & \multirow{3}{*}{$\begin{array}{l}\text { All patients } \\
N=32\end{array}$} & \multicolumn{3}{|l|}{ Disease severity } & \multirow[t]{3}{*}{$p$ value } \\
\hline & & & Mild-to-moderate & Severe & Critical & \\
\hline & & & $N=11$ & $N=10$ & $N=11$ & \\
\hline Age, median (IQR), year & $59.2(45.2,60.0)$ & $55.6(51.2,64.8)$ & $55.9(44.7-65.1)$ & $53.5(48.02-61)$ & $60.2(54.8-71.65)$ & 0.23 \\
\hline Male, no. (\%) & $10(77)$ & $24(75)$ & $8(72.7)$ & $9(90)$ & $7(63.6)$ & 0.37 \\
\hline $\begin{array}{l}\text { Median interval from first symptoms on } \\
\text { admission (IQR), days }\end{array}$ & - & $10(9-11)$ & $9(9-11)$ & $10.5(10-12)$ & $9(8-11)$ & 0.06 \\
\hline \multicolumn{7}{|l|}{ Coexisting disorder, no. (\%) } \\
\hline Any & $0(0)$ & $14(44)$ & $2(18)$ & $3(30)$ & $9(82)$ & 0.006 \\
\hline COPD & $0(0)$ & $1(3)$ & $0(0)$ & $0(0)$ & $1(9)$ & 0.37 \\
\hline Diabetes & $0(0)$ & $5(16)$ & $0(0)$ & $1(10)$ & $4(36)$ & 0.053 \\
\hline Hypertension & $0(0)$ & $10(31)$ & $2(18)$ & $2(20)$ & $6(55)$ & 0.12 \\
\hline Cardiovascular disease & $0(0)$ & $3(9)$ & $0(0)$ & $0(0)$ & $3(27)$ & 0.042 \\
\hline Cancer or hemopathy & $0(0)$ & $0(0)$ & $0(0)$ & $0(0)$ & $0(0)$ & - \\
\hline Chronic renal disease & $0(0)$ & $1(3)$ & $0(0)$ & $0(0)$ & $1(9)$ & 0.37 \\
\hline Overweight & $0(0)$ & $2(6)$ & $1(9)$ & $1(10)$ & $0(0)$ & 0.5 \\
\hline \multicolumn{7}{|l|}{ Fever on admission } \\
\hline Median temperature $(\mathrm{IQR}),{ }^{\circ} \mathrm{C}$ & - & $38.9(38.5-39.4)$ & $38.8(38.2-39.5)$ & $38.6(38.5-39.7)$ & $39.0(38.5-39.4)$ & - \\
\hline \multicolumn{7}{|l|}{ Symptoms on admission (\%) } \\
\hline Fever & - & $32(100)$ & $11(100)$ & $10(100)$ & $11(100)$ & \\
\hline Dyspnea & - & $32(100)$ & $11(100)$ & $10(100)$ & $11(100)$ & \\
\hline Cough & - & $31(97)$ & $10(91)$ & $10(100)$ & $11(100)$ & 0.37 \\
\hline Fatigue & - & $31(97)$ & $10(91)$ & $10(100)$ & $11(100)$ & 0.37 \\
\hline Myalgia & - & $20(63)$ & $8(73)$ & $8(10)$ & $4(36)$ & 0.082 \\
\hline Diarrhea & - & $11(34)$ & $4(36)$ & $5(50)$ & $1(9)$ & 0.091 \\
\hline \multicolumn{7}{|l|}{ Median oxygen requirement (IQR, L/min) } \\
\hline \multicolumn{7}{|l|}{ Clinical outcomes, no. (\%) } \\
\hline Thrombotic events & - & $3(9)$ & $0(0)$ & $1(10)$ & $2(18)$ & 0.34 \\
\hline Clinical worsening requiring MV & - & - & $0(0)$ & $5(50)$ & - & - \\
\hline Death & - & $5(15.6)$ & $0(0)$ & $0(0)$ & $5(45.5)$ & $<0.001$ \\
\hline \multicolumn{7}{|l|}{ Laboratory findings on admission } \\
\hline Leukocytes (IQR), × 109\%/L & - & $6.7(4.31-8.82)$ & $4.71(3.78-5.68)$ & $7.78(6.46-8.43)$ & $9.38(5.48-10.49)$ & 0.038 \\
\hline Neutrophils (IQR), $\times 10^{9} / \mathrm{L}$ & - & $5.08(3.12-7.37)$ & $3.25(2.07-3.44)$ & $5.81(4.74-6.36)$ & $7.69(4.32-9.13)$ & 0.022 \\
\hline Lymphocytes (IQR), × 109/L & - & $0.84(0.56-1.13)$ & $1.00(0.84-1.40)$ & $0.88(0.57-1.12)$ & $0.65(0.45-0.84)$ & 0.031 \\
\hline Monocytes (IQR), × 109/L & - & $0.41(0.23-0.52)$ & $0.40(0.26-0.52)$ & $0.42(0.27-0.51)$ & $0.33(0.12-1.05)$ & 0.95 \\
\hline Platelets (IQR), × $10^{9} / \mathrm{L}$ & - & 249 (159-298) & $166(112-251)$ & $229(170-282)$ & $313(199-352)$ & 0.007 \\
\hline CRP (IQR), mg/L & $0.7(0.0-0.8)$ & $118(55-242)$ & $30(14-76)$ & $169(136-249)$ & 159 (109-308) & $<0.001$ \\
\hline Lactate dehydrogenase (IQR), U/L & $169(155-224)$ & $424(346-574)$ & $262(196-454)$ & $411(396-623)$ & $504(426-614)$ & 0.1 \\
\hline
\end{tabular}

Significant values $(p<0.05)$ are emphasized in bold

Quantitative variables are described as median (interquartile range) and compared using the Kruskal-Wallis test; qualitative variables are described as $n$ (\%) and compared using the $X^{2}$ test of independence

COPD chronic obstructive pulmonary disease, $C R P C$-reactive protein, $I Q R$ interquartile range, $M V$ mechanical ventilation

(moderate and severe patients) also found that higher sPselectin values were associated with worst clinical outcomes, albeit without reaching significance (AUC $=0.66$, $p=0.086$ for intubation and $\mathrm{AUC}=0.70, p=0.12$ for in-hospital mortality) (Additional file 1: Figure S1). Interestingly, the predictive potential of normalized sPselectin in non-COVID-19 septic ICU patients from a pre-pandemic cohort was not significantly associated with in-hospital mortality $(\mathrm{AUC}=0.54, p=0.71)$ (Additional file 2: Figure S2).

Together, these data suggested that sP-selectin is associated with in-hospital mortality in COVID-19 patients and may identify patients at higher risk of critical illness. 


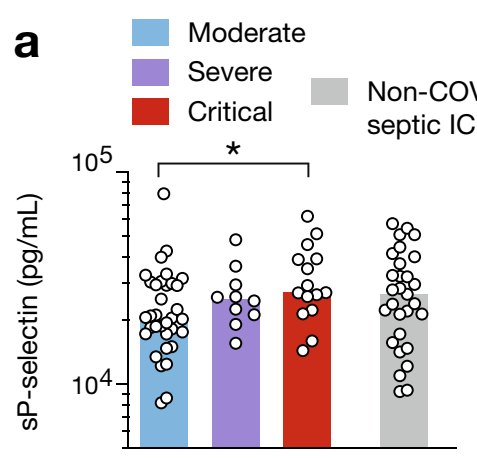

b

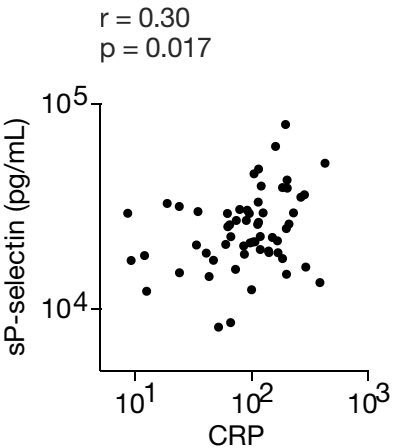

\section{C}

$$
\begin{aligned}
& r=0.10 \\
& p=0.43
\end{aligned}
$$

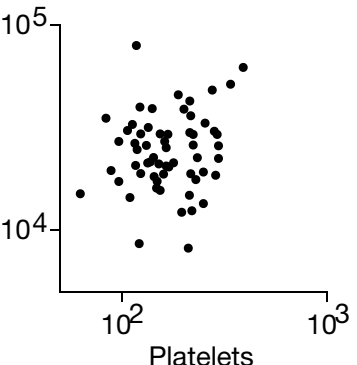

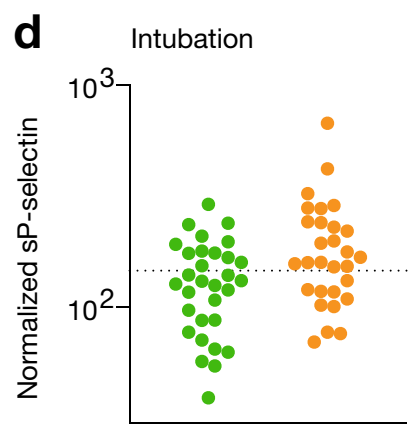
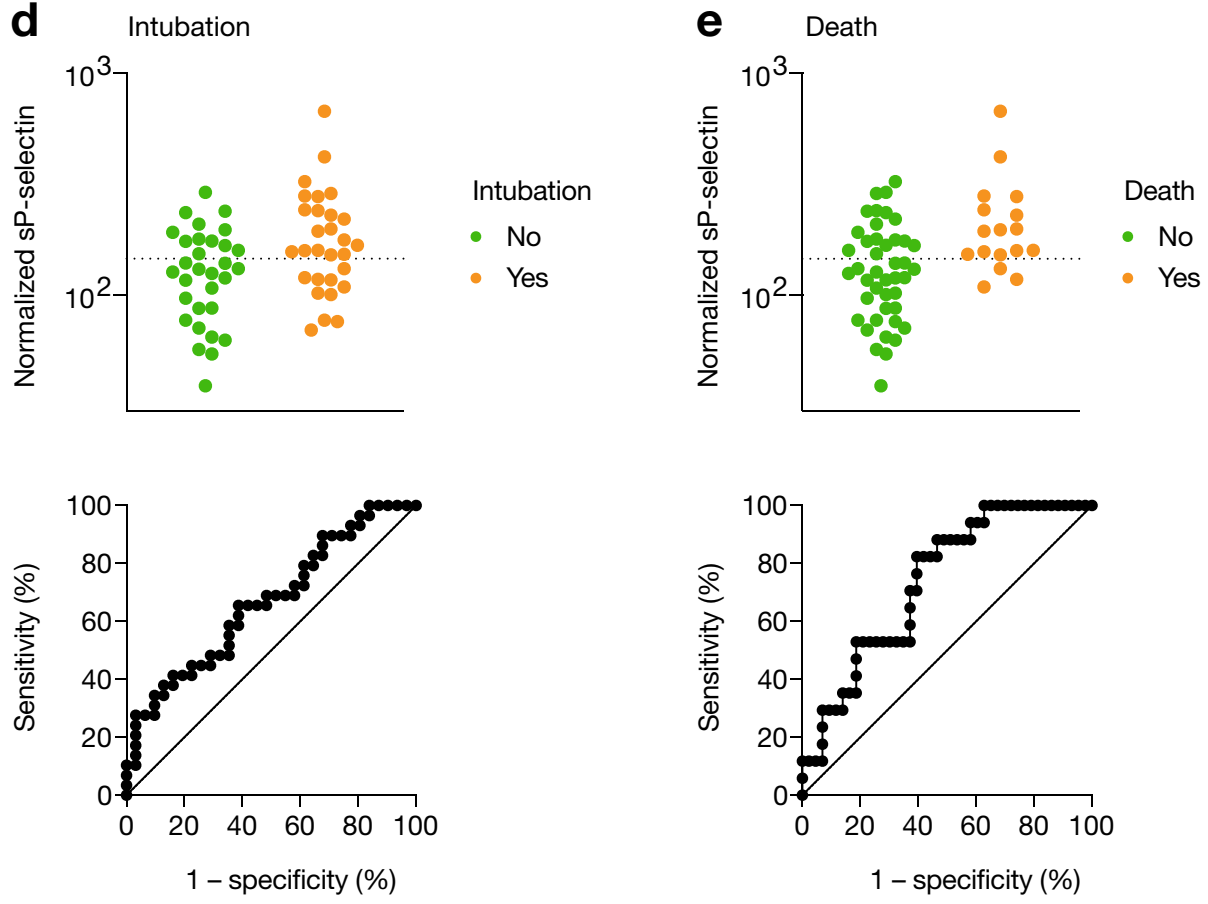

$$
\begin{gathered}
\mathrm{AUC}=0.67 \\
p=0.028
\end{gathered}
$$

Intubation

- No

- Yes

1 - specificity (\%)

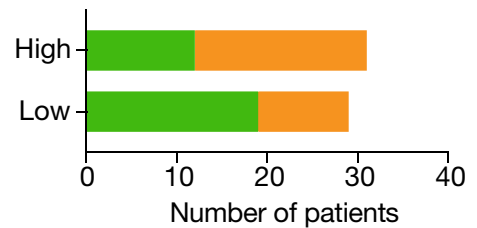

$\mathrm{AUC}=0.74$

$p=0.0047$

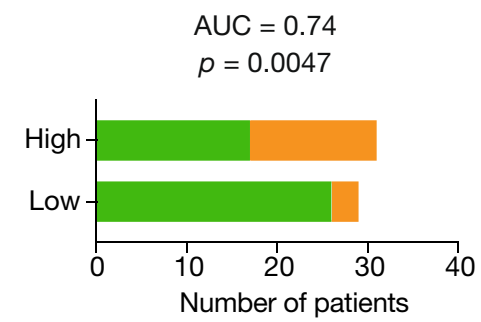

Fig. 1 sP-selectin as a marker of COVID-19 severity and a predictor of intubation and death. a Soluble P-selectin was measured in plasma from patients from COVID-19 patients from Cohort 1 and plotted according to severity grade (moderate, severe or critical disease) or from non-COVID-19 septic shock patients. Groups: mild-to-moderate $(n=35)$, severe $(n=10)$, critical $(n=15)$, non-COVID-19 septic shock $(n=29) .{ }^{*} p<0.05$ (KruskalWallis test followed by Dunn's post-test). b, c, sP-selectin correlation with CRP levels (b) or platelet counts (c). $r$ and $p$ values were determined using Spearman's correlation test. d, e, sP-selectin normalized to platelet counts as a predictor of intubation (d) or death (e). ROC curves with area under the curve (AUC) and associated $p$ values are shown. Optimal threshold was determined by maximization of Youden's index, and represented by a dashed line. Bar graphs indicate the number of patients in each group depending on normalized sP-selectin levels with respect to the optimal threshold. Groups: no intubation $(n=31)$, intubation $(n=29)$, no death $(n=43)$, death $(n=17)$ 
Critically ill COVID-19 patients display a platelet activation transcriptional signature

We previously performed a whole-blood immunological transcriptional characterization on 32 patients with laboratory-confirmed COVID-19 displaying various disease severity (cohort 2) [27]. Using an unbiased gene set enrichment analysis comparing different COVID-19 severity grades, we determined a gene signature that specifically discriminated severe from critical illness. GSEA pathway enrichment analysis identified this signature as related to primary hemostasis (Fig. 2a). Hierarchical clustering identified genes that were upregulated in a grade-dependent manner, thereby driving the primary hemostasis signature in critically ill patients (Fig. 2b). These genes included ITG2AB, GP1BB, PPBP and SELPLG (Fig. 3a), which recapitulated multiple steps of platelet activation [29, 30]. Importantly, and similarly to sP-selectin, RNA levels more strongly correlated with CRP level (Fig. 3b) than with platelet counts (Fig. 3c).

We next evaluated the ability of these genes to predict clinical outcome after normalization with platelets. Among patients that did not initially require critical care upon admission (i.e., moderate and severe patients, $n=21), 5(24 \%)$ presented respiratory failure afterwards requiring mechanical ventilation (Table 2). $P P B P$ and SELPLG were found to be the best predictor of clinical worsening (AUC $=0.8, p=0.048$ for both outcomes) (Fig. $4 \mathrm{a}-\mathrm{c}$ ). An optimal cutoff value for $P B P P$ of 5 normalized RNA counts (NRC, normalized to $10^{6}$ platelets/ $\mathrm{mL}$ ) was predictive of intubation with $100 \%$ sensitivity, $63 \%$ specificity, $100 \%$ NPV and $45 \%$ PPV, while a cutoff value of 2.65 NRC for SELPLG was predictive of intubation with $100 \%$ sensitivity, $69 \%$ specificity, $100 \%$ NPV and $50 \%$ PPV. Overall, these markers provide excellent negative predictive values, with no patient below the threshold value requiring future mechanical ventilation.

\section{Discussion}

Dysregulated hemostasis is emerging as a key factor in COVID-19 pathogenesis and severity. The present study provides new insights into the contribution of platelets to disease severity with the identification of a unique hemostasis signature in critically ill patients. We identified sPselectin as a soluble marker associated with in-hospital mortality, and elevated SELPLG and PPBP RNA levels as strong negative predictors of mechanical ventilation in hospitalized patients.

a

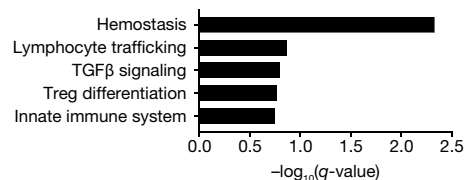

b

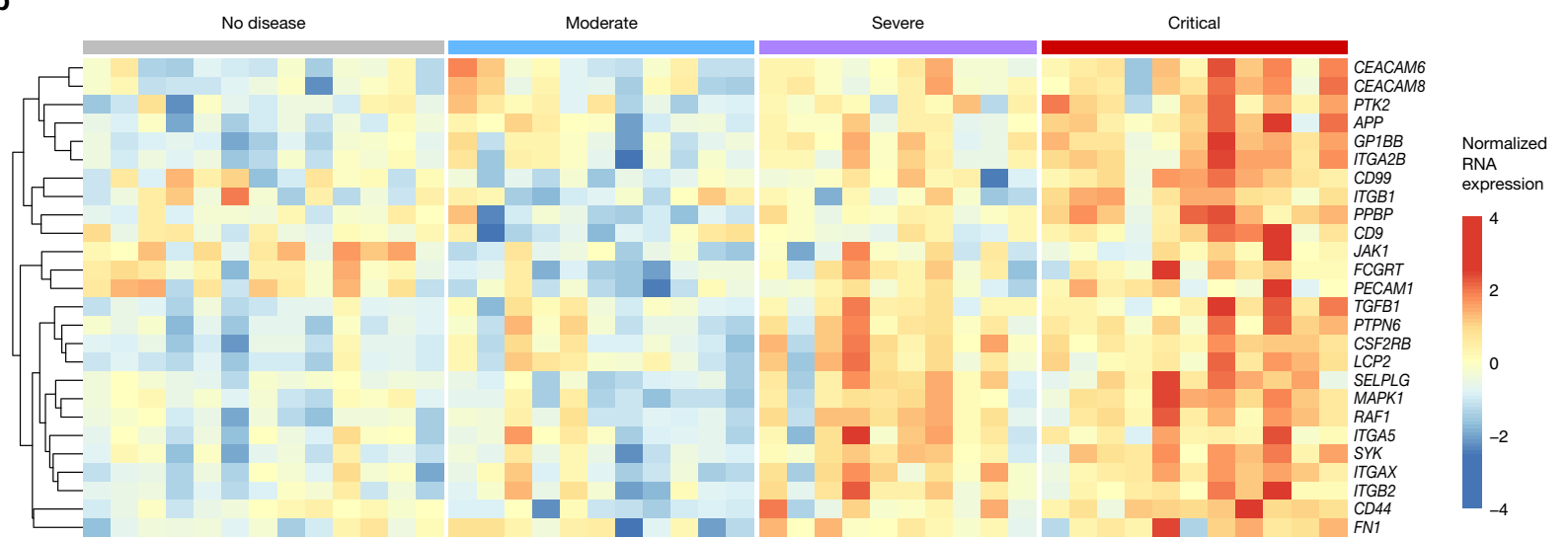

Fig. 2 Unbiased transcriptomic analysis reveals primary hemostasis-related genes as upregulated in critically ill COVID-19 patients. RNA from patients of Cohort 2 was extracted from whole blood and RNA counts of 594 genes were determined by direct probe hybridization using the Nanostring nCounter Human Immunology v2 kit. a Gene-set enrichment analysis was performed after ranking genes according to their differential expression in severe vs critical patients. Shown are pathways significantly enriched (false discovery rate $<0.2$ ). $\mathbf{b}$ Heatmap representation of genes of the primary hemostasis pathway that are upregulated in a severity grade-dependent manner (determined by hierarchical clustering). Up-regulated genes are shown in red and down-regulated genes in blue. Groups: healthy controls $(n=13)$, mild-to-moderate $(n=11)$, severe $(n=10)$ and critical $(n=11)$ 


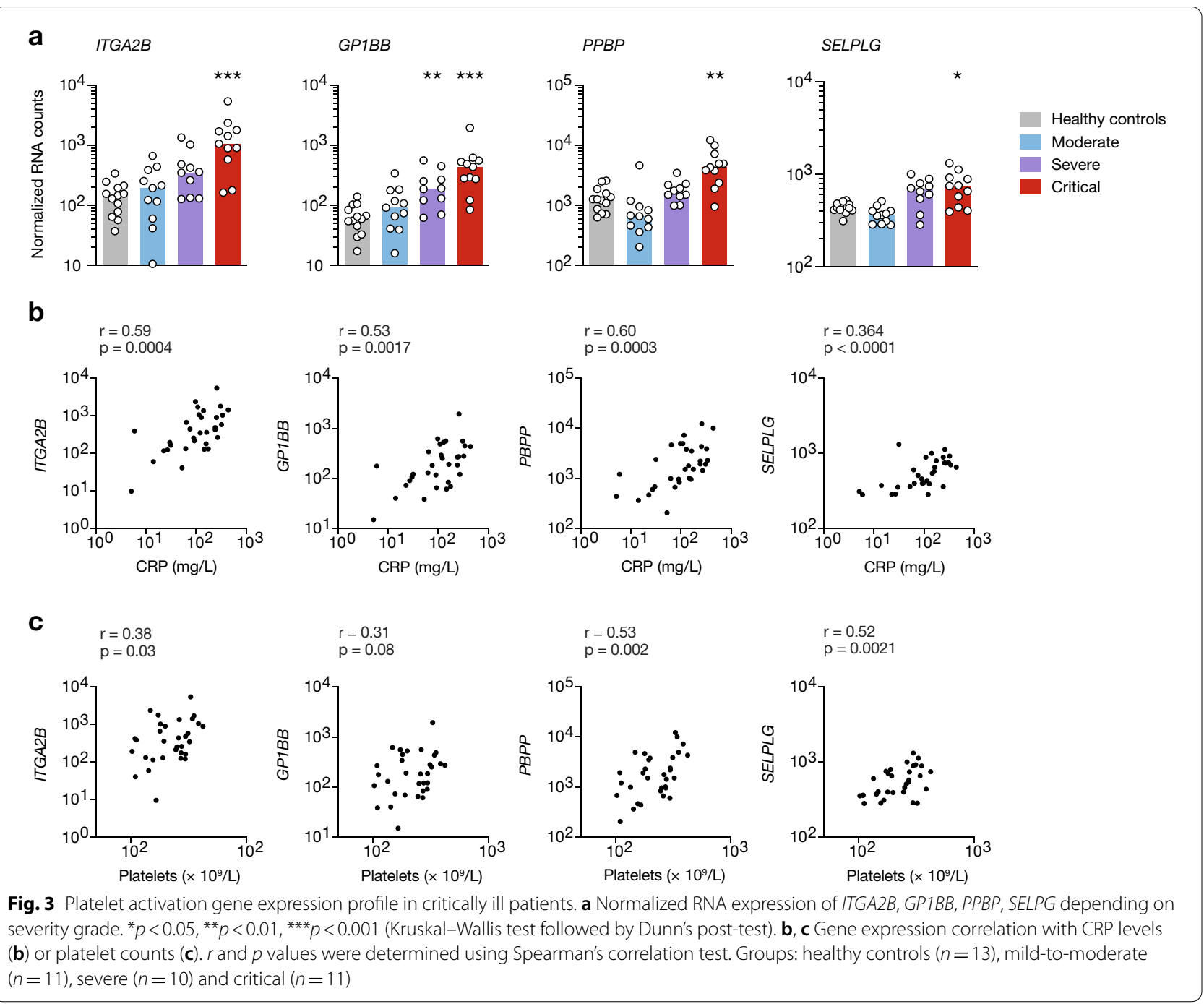

Elevated sP-selectin levels promote leukocyteendothelial and leucocyte-platelet adhesion [31], release of citrullinated histones and neutrophil extracellular traps (NETs) formation [32], promoting formation of platelet-neutrophil or -monocyte aggregates via binding of P-selectin to its ligand PSGL-1 (encoded by SELPLG) expressed on the surface of leukocytes [33]. Accordingly, elevated sP-selectin and increase in platelet-monocyte aggregates and have been recently described in COVID19 patients [33-35], the latter being effectively blocked by platelet P-selectin neutralization [33]. Our study supports these findings and highlights the key role of platelet activation in critical patients.

Importantly, while signatures related to innate immune activation and impaired interferon activity distinguished mild-to-moderate disease from severe and critical disease [27], we show that only a primary hemostasisrelated gene signature could further distinguish severe from critical patients (Fig. 2a), especially the specific platelet chemokine-encoding gene $P P B P / C X C L 7$ and SELPLG, encoding for the P-selectin glycoprotein ligand-1 (PSGL-1), which also predicted clinical outcome. Pro-platelet basic protein (PPBP), also known as CXCL7, is the most abundant platelet chemokine [29, 36], expressed within platelets as an inactive precursor and activated after cleavage during thrombus formation by enzymes released by neutrophils. PSGL-1 is expressed by leukocytes, allowing the formation of platelet-leukocyte conjugates and adhesion of leukocytes to activated endothelium through its interaction with P- or E-selectin $[29,37]$. PBPP/CXCL7 has been shown to be essential for neutrophil migration into the thrombus [38], and the formation of platelet-neutrophil aggregates and murine models of acute lung injury showed that deletion of $P P B P / C X L C 7$ protected the mice from lung disease [36], as well as blocking platelet activation [39]. Overall, our 

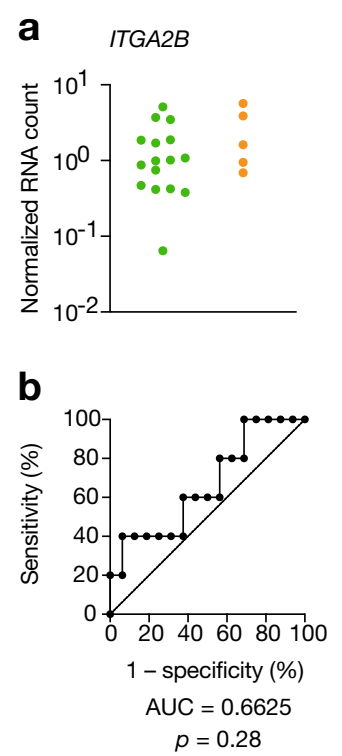
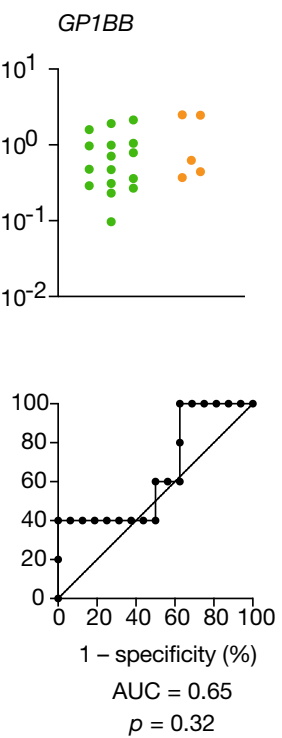

C

$$
\begin{aligned}
& \text { Intubation } \\
& \text { No } \\
& \text { Yes }
\end{aligned}
$$
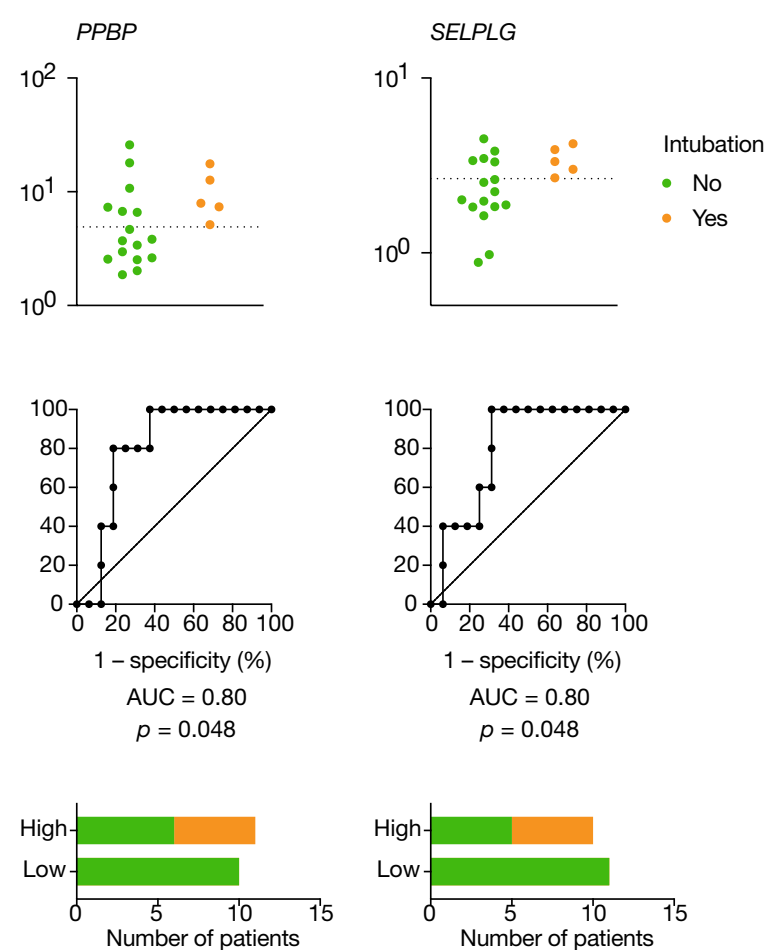

Fig. 4 Prediction potential of hemostasis genes for mechanical ventilation. a RNA levels of non-severe patients at admission depending on their future aggravation, normalized to platelet counts. Dashed lines represent optimal threshold obtained by maximization of Youden's index for significant AUCs. b Associated ROC curves, with area under the curve (AUC) and associated $p$ values. $\mathbf{c}$ Bar graphs indicating the number of patients in each group depending on normalized RNA levels of a single gene with respect to the optimal threshold. Groups: no intubation ( $n=16)$ and intubation $(n=5)$

data support a model, whereby platelet activation induces $\mathrm{PPBP} / \mathrm{CXCL7}$ release and P-selectin upregulation followed by neutrophil attraction into sites of injury and the formation of platelet-leucocyte aggregates, precipitating organ injury and failure.

In addition to their role in primary hemostasis, platelets are an integral part of the immune response to pathogens. The functional interdependence and the coordinated activation of both processes, designated as thrombo-inflammation, may drive adverse effects, such as thrombosis, multiple organ failure and death [40]. We support the hypothesis that deregulated primary hemostasis, in association to inflammation, could drive COVID-19 progression into critical disease. Viral infection and sepsis models have been associated with platelet gene expression and functional alterations [41, 42]. Supporting this generalizable thrombo-inflammation model, we found similar elevated sP-selectin in non-COVID septic ICU patients. However, some unique features may characterize SARS-CoV-2-mediated platelet dysregulation. Authors have shown that sP-selectin seemed to be more strongly associated COVID-19-related in-hospital mortality [43, 44]. Frazer et al. [45] found significantly elevated sP-selectin in ICU patients in comparison to non-COVID-19 ICU patients at day 3 post-admission (and not at days 1 and 2), that persisted until day 7. This finding suggests that persistent platelet activation may characterize COVID-19-associated coagulopathy. In addition, Manne et al. showed that platelet gene expression profile was unique in COVID-19 patients in comparison to $\mathrm{H} 1 \mathrm{~N} 1$ pandemic patients. Altogether, these data demonstrate that COVID-19 critical patients share important similarities with other critical sepsis, but some features may uniquely contribute to platelet activation during SARS-CoV-2 infection.

Prediction of illness trajectories after the onset of symptoms is difficult but remains critical. Clinical and biological factors have been shown to predict poor outcome, including arterial blood gazes values (e.g., hypoxemia, hypercapnia, $P / F$ ratio, lactate), inflammatory markers (e.g., CRP, IL-6), coagulation markers (e.g., VWF, D-dimer) and cellular blood composition (e.g., neutrophil-to-lymphocyte ratio). These factors have led to the development of prognosis scores to predict critical illness [46]. Our findings suggest that markers of dysregulated 
hemostasis could be evaluated for their predictive prognostic value.

Finally, these findings suggest that a subset of patient may benefit from drugs preventing platelet activation, such as antiplatelet agents or P-selectin inhibitors [47], which have also been proposed in other septic settings $[48,49]$. These anti-platelets agents in combination with anti-inflammatory drugs (glucocorticoids, anti-IL-6) could have substantial impact on thrombo-inflammation.

The benefit of anti-platelet agents is, however, challenged by recent findings from Althaus and coll. [50] which proposed that the induction of a procoagulant platelet subpopulation may be a key driver of critical COVID-19. Procoagulant platelets displayed increased P-selectin, integrin $\alpha \mathrm{IIb}$ and GPIb $\alpha$ expression on their surface [51], in line with biomarkers (sP-selectin, $P P B P$, SELPLG) and signature (ITG2AB, GP1BB, PPBP and $S E L P L G)$ we identified. However, in this scenario, the benefits of aspirin and $\mathrm{P} 2 \mathrm{Y}_{12}$ inhibitors would be limited; blockade of $\mathrm{P} 2 \mathrm{Y}_{1}$ and $\mathrm{P} 2 \mathrm{Y}_{12}$ does not inhibit procoagulant platelets activation [52] and pretreatment with aspirin has minimal effect on their generation [53]. In contrast, Manne et al. [35] did not find evidence for an increased procoagulant platelet subpopulation, and an additional study concluded that procoagulant platelets may, on the contrary, have a protective effect during SARS-CoV-2-related pneumonia [54]. Therefore, the mechanisms underlying platelets activation, and subsequently, the potential benefit of anti-platelet therapy remain controversial. The REMA-CAP trial is currently investigating the use of aspirin or a $\mathrm{P}_{2} \mathrm{Y}_{12}$ inhibitor (clopidogrel, prasugrel, or ticagrelor) [55], and results from the RECOVERY trial on aspirin will soon be unraveled [56]. So far, one retrospective trial suggested a potential benefit of aspirin use on critical care and in-hospital mortality [57].

Our study has some limitations. We used two independent cohorts with a small sample size from two centers. The two cohorts were not homogenous in regard to timing of measurements and severity of patients. In addition, the study was not designed as a longitudinal study, so no sequential measurement was available. Indeed, sP-selectin association with clinical outcome was moderate and serial measurement over time may optimize its outcome prediction performance. Moreover, longitudinal analysis of sP-selectin levels may provide insights on the causal role of platelets activation in driving critical disease. Our transcriptomic analysis was performed on whole-blood RNA, so we cannot evaluate the contribution of each cell population to hemostasis dysregulation. Separate transcriptional profiling of platelets vs other circulating populations and analysis of cell expression phenotypes (P-selectin, phosphatidyl-serine exposure) and evaluation of platelets functions may provide further insights into the contribution of each population to thrombo-inflammation and dissect the mechanisms of platelet-neutrophil or platelet-monocyte aggregates.

Overall, this exploratory study sheds light onto the role of thrombo-inflammation in critical patients. We identified platelet activation markers sP-selectin, SELPLG and PPBP/ $C X C L 7$ as potential biomarkers of critical worsening. Additional studies dedicated to evaluate the predictive performance of these biomarkers are required to both validate our findings and optimize their ability to predict progression to critical disease.

\section{Supplementary Information}

The online version contains supplementary material available at https://doi. org/10.1186/s13613-021-00899-1.

Additional file 1: Figure S1. sP-selectin as a marker of later requirement for mechanical ventilation or in-hospital mortality in mild-to-moderate and severe COVID-19 patients sP-selectin normalized to platelet counts as a predictor of intubation (left) or death (right). Each dot represents one patient (upper panel). ROC curves with area under the curve (AUC) and associated $p$ values are shown (lower panel). Groups: no intubation $(n=31)$, intubation $(n=14)$, no death $(n=39)$, death $(n=6)$.

Additional file 2: Figure S2. sP-selectin is not associated with death in non-COVID-19 septic ICU patients. sP-selectin normalized to platelet counts as a predictor of intubation (left) or death (right). Each dot represents one patient (upper panel). ROC curves with area under the curve (AUC) and associated $p$ values are shown (lower panel). Groups: no death $(n=13)$, death $(n=16)$.

\section{Acknowledgements}

We would like to acknowledge all nurses, technicians and physicians involved in all department involved in COVID-19 patients management in APHP.CUP hospitals, for their help in taking care of patients and including them in the study. This study was supported by the Fonds IMMUNOV, for Innovation in Immunopathology. The study was also supported by the Institut National de la Santé et de la Recherche Médicale (INSERM), by a government grant managed by the Agence National de la Recherche as part of the "Investment for the Future" program (ANR-10-IAHU-01), and by grants from the Agence National de la Recherche: ANR-flash Covid19 "AIROCovid" and "CoVarlmm". We also acknowledge funding from the Institut Pasteur for Covid19 research. J.H. is a recipient of an Institut Imagine MD-PhD fellowship program supported by the Fondation Bettencourt Schueller. L.B. is supported by the EUR G.E.N.E. (reference \#ANR-17-EURE-0013) program of the Université de Paris IdEx \#ANR18-IDEX-0001 funded by the French Government through its "Investments for the Future" program. We thank AP-HP for promotion of the SARCODO Project. We thank the unit of clinical research URC HEGP CIC-EC1418 (Natacha Nohile, Pauline Jouany and Dr Juliette Djadi-Prat) and Helene Cart-Grandjean from AP-HP for their involvement in SARCODO project. SARCODO project was supported by grants of ANR (Fondation de France) and Appel d'offre AP-HP mécénat crise COVID-19 from GHU APHP.CUP.

\section{Authors' contributions}

$\mathrm{NY}, \mathrm{JB}, \mathrm{JH}, \mathrm{DMS}$ and BT conceived and designed the study and had full access to all of the data in the study and take responsibility for the integrity of the data and the accuracy of the data analysis. NY, JB, JH, RC, AP, LB, BC, DMS and BT performed research. NY, JB, DMS and BT drafted the paper. NY, JB, NG, RC, $A P, D D, D M S$ and BT did the analysis. All authors agree to be accountable for all aspects of the work in ensuring that questions related to the accuracy or integrity of any part of the work are appropriately investigated and resolved. All authors critically revised the manuscript for important intellectual content, read and approved final manuscript. 


\section{Availability of data and materials}

All data are available upon reasonable request.

\section{Declarations}

\section{Ethics approval and consent to participate}

Both studies conformed to the principles outlined in the Declaration of Helsinki, and received approval by the appropriate Institutional Review Board (CPP2020-04-048/2020-A01048-31/ 20.04.21.49318).

\section{Competing interests}

None of the authors has a relevant competing interest.

\section{Author details}

${ }^{1}$ Translational Immunology Lab, Department of Immunology, Institut Pasteur, 75015 Paris, France. ${ }^{2}$ Department of Internal Medicine, National Reference Center for Rare Systemic Autoimmune Diseases, AP-HP, APHP.CUP, Hôpital Cochin, 75014 Paris, France. ${ }^{3}$ Imagine Institute Laboratory of Immunogenetics of Pediatric Autoimmune Diseases, INSERM, UMR 1163, Université de Paris, 75015 Paris, France. ${ }^{4}$ Innovative Therapies in Haemostasis, INSERM, Université de Paris, 75006 Paris, France. ${ }^{5}$ Hematology Department, APHPCUP, 75015 Paris, France. ${ }^{6}$ Biosurgical Research Lab (Carpentier Foundation), Georges Pompidou European Hospital, 75015 Paris, France. ${ }^{7}$ Cytometry and Biomarkers UTechS, CRT, Institut Pasteur, 75015 Paris, France. ${ }^{8}$ Department of Pulmonology, APHP-CUP, Hôpital Cochin, 75014 Paris, France. ${ }^{9}$ Université de Paris, Institut Cochin, INSERM U1016, CNRS UMR8104, 75006 Paris, France. ${ }^{10}$ Service de Médecine Intensive and Réanimation, APHP-CUP, Hôpital Cochin, 75014 Paris, France. ${ }^{11}$ Vascular Medicine Department, APHP-CUP, Université de Paris, 75015 Paris, France. ${ }^{12}$ Université de Paris, INSERM, U970, PARCC, Paris, France. ${ }^{13}$ Intensive Care Unit, APHP-CUP, 75015 Paris, France. ${ }^{14}$ Emergency Department, APHP-CUP, 75015 Paris, France. ${ }^{15}$ Equipe Mobile d'Infectiologie, APHP-CUP, Hôpital Cochin, 75014 Paris, France. ${ }^{16}$ Epidemiology and Modelling of Antibiotic Evasion (EMAE), Institut Pasteur, 75015 Paris, France. ${ }^{17}$ Université de Paris, INSERM, IAME, Université de Paris, 75006 Paris, France. ${ }^{18}$ Department of Internal Medicine, Hôpital Cochin, 27, Rue du Faubourg Saint-Jacques, 75679 Paris Cedex 14, France.

Received: 25 February 2021 Accepted: 2 July 2021

Published online: 17 July 2021

\section{References}

1. Smadja DM, Mentzer SJ, Fontenay M, Laffan MA, Ackermann M, Helms J, Jonigk D, Chocron R, Pier GB, Gendron N, Pons S, Diehl JL, Margadant C, Guerin C, Huijbers EJM, Philippe A, Chapuis N, Nowak-Sliwinska P, Karagiannidis C, Sanchez O, Kümpers P, Skurnik D, Randi AM, Griffioen AW. COVID-19 is a systemic vascular hemopathy: insight for mechanistic and clinical aspects. Angiogenesis. 2021;28:1-34. https://doi.org/10.1007/ s10456-021-09805-6.

2. Huang C, Wang Y, Li X, Ren L, Zhao J, Hu Y, et al. Clinical features of patients infected with 2019 novel coronavirus in Wuhan, China. Lancet. 2020:395:497-506.

3. Salje H, Kiem CT, Lefrancq N, Courtejoie N, Bosetti P, Paireau J, et al. Estimating the burden of SARS-CoV-2 in France. Science. 2020;369:208-11.

4. Wang D, Hu B, Hu C, Zhu F, Liu X, Zhang J, et al. Clinical characteristics of 138 hospitalized patients with 2019 novel coronavirus-infected pneumonia in Wuhan, China. JAMA. 2020;323:1061.

5. Williamson EJ, Walker AJ, Bhaskaran K, Bacon S, Bates C, Morton CE, et al. Factors associated with COVID-19-related death using OpenSAFELY. Nature. 2020:584:430-6.

6. Julien P, Julien G, Morgan C, Erika P, Thibault D, Fanny L, et al. Pulmonary embolism in patients with COVID-19. Circulation. 2020;142:184-6.

7. Llitjos J, Leclerc M, Chochois C, Monsallier J, Ramakers M, Auvray M, et al. High incidence of venous thromboembolic events in anticoagulated severe COVID-19 patients. J Thromb Haemost. 2020. https://doi.org/10. $1111 /$ jth. 14869 .

8. Tang N, Bai H, Chen X, Gong J, Li D, Sun Z. Anticoagulant treatment is associated with decreased mortality in severe coronavirus disease 2019 patients with coagulopathy. J Thromb Haemost. 2020;18:1094-9.
9. Panigada M, Bottino N, Tagliabue P, Grasselli G, Novembrino C, Chantarangkul $V$, et al. Hypercoagulability of COVID-19 patients in intensive care unit: a report of thromboelastography findings and other parameters of hemostasis. J Thromb Haemost. 2020;18:1738-42.

10. Philippe A, Gendron N, Bory O, Beauvais A, Mirault T, Planquette B, Sanchez O, Diehl JL, Chocron R, Smadja DM. Von Willebrand factor collagen-binding capacity predicts in-hospital mortality in COVID-19 patients: insight from WWF/ADAMTS13 ratio imbalance. Angiogenesis. 2021:11:1-5. https://doi.org/10.1007/s10456-021-09789-3.

11. Smadja DM, Guerin CL, Chocron R, Yatim N, Boussier J, Gendron N, Khider L, Hadjadj J, Goudot G, Debuc B, Juvin P, Hauw-Berlemont C, Augy JL, Peron N, Messas E, Planquette B, Sanchez O, Charbit B, Gaussem P, Duffy D, Terrier B, Mirault T, Diehl JL. Angiopoietin-2 as a marker of endothelial activation is a good predictor factor for intensive care unit admission of COVID-19 patients. Angiogenesis. 2020;23(4):611-20. https://doi.org/10. 1007/s10456-020-09730-0

12. Carsana L, Sonzogni A, Nasr A, Rossi RS, Pellegrinelli A, Zerbi P, et al. Pulmonary post-mortem findings in a series of COVID-19 cases from northern Italy: a two-centre descriptive study. Lancet Infect Dis. 2020;20:1135-40.

13. Ackermann M, Verleden SE, Kuehnel M, Haverich A, Welte T, Laenger F, et al. Pulmonary vascular endothelialitis, thrombosis, and angiogenesis in COVID-19. N Engl J Med. 2020;383:120-8.

14. Diehl J-L, Peron N, Chocron R, Debuc B, Guerot E, Hauw-Berlemont C, et al. Respiratory mechanics and gas exchanges in the early course of COVID-19 ARDS: a hypothesis-generating study. Ann Intensive Care. 2020;10:95.

15. Thachil J, Tang N, Gando S, Falanga A, Cattaneo M, Levi M, et al. ISTH interim guidance on recognition and management of coagulopathy in COVID-19. J Thromb Haemost. 2020;18:1023-6.

16. Antithrombotic Therapy. COVID-19 Treat. Guidel. https://www.covid19tre atmentguidelines.nih.gov/antithrombotic-therapy/. Accessed 21 Feb 2021.

17. Helms J, Tacquard C, Severac F, Leonard-Lorant I, Ohana M, Delabranche $X$, et al. High risk of thrombosis in patients with severe SARS-CoV-2 infection: a multicenter prospective cohort study. Intensive Care Med. 2020;46:1089-98.

18. Helms J, Severac F, Merdji H, Schenck M, Clere-Jehl R, Baldacini M, et al. Higher anticoagulation targets and risk of thrombotic events in severe COVID-19 patients: bi-center cohort study. Ann Intensive Care. $2021 ; 11: 14$

19. Khider L, Gendron N, Goudot G, Chocron R, Hauw-Berlemont C, Cheng C, et al. Curative anticoagulation prevents endothelial lesion in COVID-19 patients. J Thromb Haemost. 2020;18:2391-9.

20. Pons S, Fodil S, Azoulay E, Zafrani L. The vascular endothelium: the cornerstone of organ dysfunction in severe SARS-CoV-2 infection. Crit Care. 2020;24:353.

21. Varga Z, Flammer AJ, Steiger $P$, Haberecker M, Andermatt R, Zinkernagel AS, et al. Endothelial cell infection and endotheliitis in COVID-19. Lancet. 2020;395:1417-8.

22. Lisman T. Platelet-neutrophil interactions as drivers of inflammatory and thrombotic disease. Cell Tissue Res. 2018;371:567-76.

23. Latrémouille C, Carpentier A, Leprince P, Roussel J-C, Cholley B, Boissier $E$, et al. A bioprosthetic total artificial heart for end-stage heart failure: results from a pilot study. J Heart Lung Transplant. 2018;37:33-7.

24. Sakamaki F, Ishizaka A, Handa M, Fujishima S, Urano T, Sayama K, et al. Soluble form of P-selectin in plasma is elevated in acute lung injury. Am J Respir Crit Care Med. 1995;151:1821-6.

25. Smadja DM, Guerin CL, Chocron R, Yatim N, Boussier J, Gendron N, et al. Angiopoietin-2 as a marker of endothelial activation is a good predictor factor for intensive care unit admission of COVID-19 patients. Angiogenesis. 2020;23:611-20.

26. Borgel D, Clauser S, Bornstain C, Bièche I, Bissery A, Remones V, et al. Elevated growth-arrest-specific protein 6 plasma levels in patients with severe sepsis. Crit Care Med. 2006;34:219-22.

27. Borgel D, Bornstain C, Reitsma PH, Lerolle N, Gandrille S, Dali-Ali F, et al. A Comparative study of the protein C pathway in septic and nonseptic patients with organ failure. Am J Respir Crit Care Med. 2007;176:878-85.

28. Hadjadj J, Yatim N, Barnabei L, Corneau A, Boussier J, Smith N, et al. Impaired type I interferon activity and inflammatory responses in severe COVID-19 patients. Science. 2020;369:718-24. 
29. Rayes J, Bourne JH, Brill A, Watson SP. The dual role of platelet-innate immune cell interactions in thrombo-inflammation. Res Pract Thromb Haemost. 2020;4:23-35.

30. Periayah MH, Halim AS, Mat Saad AZ. Mechanism action of platelets and crucial blood coagulation pathways in hemostasis. Int J Hematol-Oncol Stem Cell Res. 2017;11:319-27.

31. Woollard KJ, Andreas S, Harris EE, Eisenhardt SU, Jackson SP, Karlheinz P, et al. Pathophysiological levels of soluble P-selectin mediate adhesion of leukocytes to the endothelium through Mac-1 activation. Circ Res. 2008;103:1128-38.

32. Panicker SR, Mehta-D'souza P, Zhang N, Klopocki AG, Shao B, McEver RP. Circulating soluble P-selectin must dimerize to promote inflammation and coagulation in mice. Blood. 2017;130:181-91.

33. Hottz ED, Azevedo-Quintanilha IG, Palhinha L, Teixeira L, Barreto EA, Pão $\mathrm{CRR}$, et al. Platelet activation and platelet-monocyte aggregate formation trigger tissue factor expression in patients with severe COVID-19. Blood. 2020;136:1330-41.

34. Goshua G, Pine AB, Meizlish ML, Chang $C-H$, Zhang $H$, Bahel P, et al. Endotheliopathy in COVID-19-associated coagulopathy: evidence from a single-centre, cross-sectional study. Lancet Haematol. 2020;7:e575-82.

35. Manne BK, Denorme F, Middleton EA, Portier I, Rowley JW, Stubben C, et al. Platelet gene expression and function in patients with COVID-19. Blood. 2020;136:1317-29.

36. Bdeir K, Gollomp K, Stasiak M, Mei J, Papiewska-Pajak I, Zhao G, et al. Platelet-specific chemokines contribute to the pathogenesis of acute lung injury. Am J Respir Cell Mol Biol. 2017;56:261-70.

37. Furie B, Furie BC. Role of platelet P-selectin and microparticle PSGL-1 in thrombus formation. Trends Mol Med. 2004;10:171-8.

38. Gleissner CA, von Hundelshausen P, Ley K. Platelet chemokines in vascular disease. Arterioscler Thromb Vasc Biol. 2008;28:1920-7.

39. Zarbock A, Polanowska-Grabowska RK, Ley K. Platelet-neutrophil-interactions: linking hemostasis and inflammation. Blood Rev. 2007;21:99-111.

40. Blood platelets and sepsis pathophysiology: a new therapeutic prospect in critically [corrected] ill patients? PubMed. https://pubmed.ncbi.nlm.nih. gov/29192366/. Accessed 21 Feb 2021.

41. Campbell RA, Schwertz H, Hottz ED, Rowley JW, Manne BK, Washington $A V$, et al. Human megakaryocytes possess intrinsic antiviral immunity through regulated induction of IFITM3. Blood. 2019;133:2013-26.

42. Middleton EA, Weyrich AS, Zimmerman GA. Platelets in pulmonary immune responses and inflammatory lung diseases. Physiol Rev. 2016;96:1211-59.

43. Barrett TJ, Lee AH, Xia Y, Lin LH, Black M, Cotzia P, et al. Platelet and vascular biomarkers associate with thrombosis and death in coronavirus disease. Circ Res. 2020;127:945-7.
44. Vassiliou AG, Keskinidou C, Jahaj E, Gallos P, Dimopoulou I, Kotanidou A, et al. ICU admission levels of endothelial biomarkers as predictors of mortality in critically ill COVID-19 patients. Cells. 2021;10:186.

45. Fraser DD, Patterson EK, Slessarev M, Gill SE, Martin C, Daley M, et al. Endothelial injury and glycocalyx degradation in critically ill coronavirus disease 2019 patients: implications for microvascular platelet aggregation. Crit Care Explor. 2020;2: e0194.

46. Liang W, Liang H, Ou L, Chen B, Chen A, Li C, et al. Development and validation of a clinical risk score to predict the occurrence of critical illness in hospitalized patients with COVID-19. JAMA Intern Med. 2020;180:1081.

47. Neri T, Nieri D, Celi A. P-selectin blockade in COVID-19-related ARDS. Am J Physiol-Lung Cell Mol Physiol. 2020;318:237-8.

48. Ouyang Y, Wang Y, Liu B, Ma X, Ding R. Effects of antiplatelet therapy on the mortality rate of patients with sepsis: a meta-analysis. J Crit Care. 2019;50:162-8.

49. Akinosoglou K, Alexopoulos D. Use of antiplatelet agents in sepsis: a glimpse into the future. Thromb Res. 2014. https://doi.org/10.1016/j. thromres.2013.07.002.

50. Althaus K, Marini I, Zlamal J, Pelzl L, Singh A, Häberle H, et al. Antibodyinduced procoagulant platelets in severe COVID-19 infection. Blood. 2021;137:1061-71.

51. Fager AM, Wood JP, Bouchard BA, Feng P, Tracy PB. Properties of procoagulant platelets. Arterioscler Thromb Vasc Biol. 2010;30:2400-7.

52. Agbani EO, Williams CM, Hers I, Poole AW. Membrane ballooning in aggregated platelets is synchronised and mediates a surge in microvesiculation. Sci Rep. 2017;7:2770.

53. Hua VM, Abeynaike L, Glaros E, Campbell H, Pasalic L, Hogg PJ, et al. Necrotic platelets provide a procoagulant surface during thrombosis. Blood. 2015;126:2852-62.

54. Denorme F, Manne BK, Portier I, Petrey AC, Middleton EA, Kile BT, et al. COVID-19 patients exhibit reduced procoagulant platelet responses. J Thromb Haemost. 2020;18:3067-73.

55. REMAP-CAP Trial [Internet]. REMAP-CAP Trial. https://www.remapcap.org. Accessed 23 May 2021.

56. McCall B. Data, data all around. Lancet Digit Health. 2021;3:e284-5.

57. Chow JH, Khanna AK, Kethireddy S, Yamane D, Levine A, Jackson AM, et al. Aspirin use is associated with decreased mechanical ventilation, intensive care unit admission, and in-hospital mortality in hospitalized patients with coronavirus disease 2019. Anesth Analg. 2021;132:930-41.

\section{Publisher's Note}

Springer Nature remains neutral with regard to jurisdictional claims in published maps and institutional affiliations.

\section{Submit your manuscript to a SpringerOpen ${ }^{\circ}$ journal and benefit from:}

- Convenient online submission

- Rigorous peer review

- Open access: articles freely available online

- High visibility within the field

- Retaining the copyright to your article

Submit your next manuscript at $\boldsymbol{\nabla}$ springeropen.com 